\title{
An Efficient Hybrid Conjugate Gradient Method with the Strong Wolfe-Powell Line Search
}

\author{
Ahmad Alhawarat, ${ }^{1}$ Mustafa Mamat, ${ }^{2}$ Mohd Rivaie, ${ }^{3}$ and Zabidin Salleh ${ }^{1}$ \\ ${ }^{1}$ School of Informatics and Applied Mathematics, Universiti Malaysia Terengganu, 21030 Kuala Terengganu, Terengganu, Malaysia \\ ${ }^{2}$ Faculty of Informatics and Computing, Universiti Sultan Zainal Abidin, 21300 Kuala Terengganu, Terengganu, Malaysia \\ ${ }^{3}$ Department of Computer Science and Mathematics, Universiti Teknologi MARA (UITM) Terengganu, Campus Kuala Terengganu, \\ 21080 Kuala Terengganu, Terengganu, Malaysia
}

Correspondence should be addressed to Ahmad Alhawarat; abadee2010@yahoo.com

Received 11 March 2015; Revised 6 July 2015; Accepted 7 July 2015

Academic Editor: Haipeng Peng

Copyright ( 2015 Ahmad Alhawarat et al. This is an open access article distributed under the Creative Commons Attribution License, which permits unrestricted use, distribution, and reproduction in any medium, provided the original work is properly cited.

Conjugate gradient (CG) method is an interesting tool to solve optimization problems in many fields, such as design, economics, physics, and engineering. In this paper, we depict a new hybrid of CG method which relates to the famous Polak-Ribière-Polyak (PRP) formula. It reveals a solution for the PRP case which is not globally convergent with the strong Wolfe-Powell (SWP) line search. The new formula possesses the sufficient descent condition and the global convergent properties. In addition, we further explained about the cases where PRP method failed with SWP line search. Furthermore, we provide numerical computations for the new hybrid CG method which is almost better than other related PRP formulas in both the number of iterations and the CPU time under some standard test functions.

\section{Introduction}

The nonlinear conjugate gradient (CG) method is a useful tool to find the minimum value for unconstrained optimization problems. Consider the following form:

$$
\min \left\{f(x) \mid x \in R^{n}\right\},
$$

where $f: R^{n} \rightarrow R$ is continuously differentiable function and its gradient is denoted by $g(x)=\nabla f(x)$. The CG method is to find a sequence of points $x_{k}, k \geq 1$, starting from initial point $x_{1} \in R^{n}$ which is given as the following iterative formula:

$$
x_{k+1}=x_{k}+\alpha_{k} d_{k},
$$

where $x_{k}$ is the current iteration point and $\alpha_{k}>0$ is the step length obtained by some line search. The search direction $d_{k}$ is defined by

$$
d_{k}= \begin{cases}-g_{k}, & k=1, \\ -g_{k}+\beta_{k} d_{k-1}, & k>1,\end{cases}
$$

where $g_{k}=g\left(x_{k}\right)$ and $\beta_{k}$ is known as CG method, formula, or coefficient.

To find the step length $\left(\alpha_{k}\right)$ we can use the exact line search which is given by

$$
f\left(x_{k}+\alpha_{k} d_{k}\right)=\min f\left(x_{k}+\alpha d_{k}\right), \quad \alpha \geq 0 .
$$

In fact, (4) is not an effective line search since it needs heavy computations in function and gradient evaluations. Therefore, we prefer to use inexpensive line search. The strong Wolfe-Powell (SWP) line search $[1,2]$, which is given as follows,

$$
\begin{aligned}
f\left(x_{k}+\alpha_{k} d_{k}\right) & \leq f\left(x_{k}\right)+\delta \alpha_{k} g_{k}^{T} d_{k}, \\
\left|g\left(x_{k}+\alpha_{k} d_{k}\right)^{T} d_{k}\right| & \leq-\sigma\left|g_{k}^{T} d_{k}\right|,
\end{aligned}
$$

where $0<\delta<\sigma<1$, is to find an approximation of $\alpha_{k}$ where the descent property (see (14)) must be satisfied and no longer searching in the direction when $x_{k}$ is far from the solution. Thus, by using SWP line search we inherit the advantages of exact line search with inexpensive and low computational 
cost. However, different choices of $\alpha_{k}$ and $d_{k}$ imply different CG methods. In fact the SWP line search is a strong version of weak Wolfe-Powell (WWP) line search where the latter is given by (5) and

$$
g\left(x_{k}+\alpha_{k} d_{k}\right)^{T} d_{k} \geq \sigma g_{k}^{T} d_{k}
$$

The CG method has been developed recently based on its simplicity, numerical efficiency, and low memory requirements. Thus, it is used widely in engineering medical science and other fields. As an application in engineering we can use CG method to solve some real life problem similar to that mentioned in [3]. The CG method is limited for the functions where their gradient is available. Thus, the heuristic algorithm [4] can be used as an alternative method to find the solution for general functions. A heuristic algorithm is to find an approximation solution for the objective functions with accepted time. In addition, the heuristic algorithms can be applied without using computers. We refer the reader to see some applications for this algorithm in [5-7].

The most popular formulas for $\beta_{k}$ are Hestenes-Stiefel (HS) [8], Fletcher-Reeves (FR) [9], Polak-Ribière-Polyak (PRP) [10], and Wei et al. (WYL) [11], respectively, as follows:

$$
\begin{aligned}
\beta_{k}^{\mathrm{HS}} & =\frac{g_{k}^{T}\left(y_{k-1}\right)}{d_{k-1}^{T} y_{k-1}}, \\
\beta_{k}^{\mathrm{FR}} & =\frac{\left\|g_{k}\right\|^{2}}{\left\|g_{k-1}\right\|^{2}}, \\
\beta_{k}^{\mathrm{PRP}} & =\frac{g_{k}^{T} y_{k-1}}{\left\|g_{k-1}\right\|^{2}}, \\
\beta_{k}^{\mathrm{WYL}} & =\frac{g_{k}^{T}\left(g_{k}-\left(\left\|g_{k}\right\| /\left\|g_{k-1}\right\|\right) g_{k-1}\right)}{\left\|g_{k-1}\right\|^{2}},
\end{aligned}
$$

where $y_{k-1}=g_{k}-g_{k-1}$.

Hestenes-Stiefel [8] proposed the first formula for solving the quadratic functions in 1952. Fletcher and Reeves [9] presented the first formula (9) for nonlinear functions in 1964. The convergence properties of FR method with exact line search were obtained by Zoutendijk [12]. Al-Baali [13] proved that FR method is globally convergent with the SWP line search when $\sigma<1 / 2$. Later on Guanghui et al. [14] extended the result to $\sigma \leq 1 / 2$. The global convergence of PRP method (10) with the exact line search was proved by Elijah and Ribiere in [10]. Powell [15] gave out a counterexample showing that there exists nonconvex function, where PRP method does not converge globally, even when the exact line search is used. Powell suggested using nonnegative PRP method to reveal this problem. Gilbert and Nocedal [16] proved that nonnegative $\mathrm{PRP}(\mathrm{PRP}+)$ method, that is, $\beta_{k}=$ $\max \left\{\beta_{k}^{\mathrm{PRP}}, 0\right\}$, is globally convergent under complicated line searches. However, there is no guarantee that PRP+ is convergent with SWP line search for general nonlinear functions.
Touati-Ahmed and Storey [17] suggest the following hybrid method:

$$
\beta_{k}^{\mathrm{TS}}= \begin{cases}\beta_{k}^{\mathrm{PRP}}, & \text { if } 0 \leq \beta_{k}^{\mathrm{PRP}} \leq \beta_{k}^{\mathrm{FR}} \\ \beta_{k}^{\mathrm{FR}}, & \text { otherwise. }\end{cases}
$$

In 2006 Wei et al. [11] presented a new positive CG method (11), which is quite similar to original PRP method which has been studied in both exact and inexact line search. Many modifications have appeared, such as the following [18-20], respectively:

$$
\begin{aligned}
\beta_{k}^{\mathrm{VHS}} & =\frac{\left\|g_{k}\right\|^{2}-\left(\left\|g_{k}\right\| /\left\|g_{k-1}\right\|\right) g_{k} g_{k-1}}{d_{k-1}^{T} y_{k-1}}, \\
\beta_{k}^{\mathrm{NPRP}} & =\frac{\left\|g_{k}\right\|^{2}-\left(\left\|g_{k}\right\| /\left\|g_{k-1}\right\|\right)\left|g_{k} g_{k-1}\right|}{\left\|g_{k-1}\right\|^{2}}, \\
\beta_{k}^{\mathrm{DPRP}} & =\frac{\left\|g_{k}\right\|^{2}-\left(\left\|g_{k}\right\| /\left\|g_{k-1}\right\|\right)\left|g_{k} g_{k-1}\right|}{m\left|g_{k}^{T} d_{k-1}\right|+\left\|g_{k-1}\right\|^{2}},
\end{aligned}
$$

where $m \geq 1$.

Recently many CG formulas were constructed in order to get the efficiency and robustness. For more about the latest CG methods we refer the reader to see $[21,22]$.

One of the important rules in CG methods is the descent condition; that is, if one can prove

$$
g_{k}^{T} d_{k}<0
$$

then we have a guarantee for $f\left(x_{k+1}\right)<f\left(x_{k}\right)$. If we extended (14) to the following form,

$$
g_{k}^{T} d_{k} \leq-c\left\|g_{k}\right\|^{2}, \quad k \geq 0, c>0,
$$

then (15) is called the sufficient descent condition.

This paper is organized as follows; in Section 2 we will present the current problem for PRP and nonnegative PRP method with SWP line search. Later on we will suggest the new hybrid CG formula and its simplifications. Furthermore, we will establish the global convergence properties with the SWP line search, in Section 3. Numerical results with conclusion will be presented in Sections 4 and 5, respectively.

\section{Motivation and the Hybrid Formula}

The PRP formula is one of the best CG methods in this century. However, as we mentioned before this method fails to solve some standard test problems for nonconvex functions; even the exact line search is used. Thus, the main contribution of this paper is to extend using PRP formula in several cases with SWP line search under mild condition and restart the CG algorithm by using NPRP CG formula when PRP failed to satisfy that condition.

The following discussion illustrates the cases in which PRP method fails and succeeds with SWP line search to 
obtain the convergence properties. The PRP method could be simplified as follows:

$$
\beta_{k}^{\mathrm{PRP}}=\frac{g_{k}^{T}\left(g_{k}-g_{k-1}\right)}{\left\|g_{k-1}\right\|^{2}}=\frac{\left\|g_{k}\right\|^{2}-g_{k}^{T} g_{k-1}}{\left\|g_{k-1}\right\|^{2}} .
$$

Therefore we have the following cases.

Case A. If $g_{k}^{T} g_{k-1}>0$, then we have the following two possibilities.

Case A1. If $\left\|g_{k}\right\|^{2}>g_{k}^{T} g_{k-1}$, then we have $0 \leq \beta_{k}^{\mathrm{PRP}} \leq \beta_{k}^{\mathrm{FR}}$. In this case, PRP method is efficient and has global convergence properties.

Case A2. If $\left\|g_{k}\right\|^{2}<g_{k}^{T} g_{k-1}$, then we have $\beta_{k}^{\text {PRP }}<0$. In this case based on [16] we fail to obtain the global convergence properties for nonconvex functions.

Case B. If $g_{k}^{T} g_{k-1}<0$, then

$$
\begin{aligned}
\beta_{k}^{\mathrm{PRP}} & =\frac{g_{k}^{T}\left(g_{k}-g_{k-1}\right)}{\left\|g_{k-1}\right\|^{2}}=\frac{\left\|g_{k}\right\|^{2}+\left|g_{k}^{T} g_{k-1}\right|}{\left\|g_{k-1}\right\|^{2}} \\
& =\beta_{k}^{\mathrm{FR}}+\frac{\left|g_{k}^{T} g_{k-1}\right|}{\left\|g_{k-1}\right\|^{2}} .
\end{aligned}
$$

$$
\beta_{k}^{\mathrm{PRP}^{* *}}= \begin{cases}\beta_{k}^{\mathrm{PRP}}=\frac{g_{k}^{T}\left(g_{k}-g_{k-1}\right)}{\left\|g_{k-1}\right\|^{2}}, & \text { if }\left\|g_{k}\right\|^{2}>\left|g_{k}^{T} g_{k-1}\right|, \\ \beta_{k}^{\mathrm{NPRP}}=\frac{\left\|g_{k}\right\|^{2}-\left(\left\|g_{k}\right\| /\left\|g_{k-1}\right\|\right)\left|g_{k} g_{k-1}\right|}{\left\|g_{k-1}\right\|^{2}}, & \text { otherwise. }\end{cases}
$$

If $\beta_{k}^{\mathrm{PRP}^{* *}}=\left(\left\|g_{k}\right\|^{2}-g_{k}^{T} g_{k-1}\right) /\left\|g_{k-1}\right\|^{2}$, then under the condition $\left\|g_{k}\right\|^{2}>\left|g_{k}^{T} g_{k-1}\right|$, we obtain

$$
\begin{aligned}
0 & <\beta_{k}^{\mathrm{PRP}^{* *}}=\frac{\left\|g_{k}\right\|^{2}-g_{k}^{T} g_{k-1}}{\left\|g_{k-1}\right\|^{2}} \leq \frac{\left\|g_{k}\right\|^{2}+\left|g_{k}^{T} g_{k-1}\right|}{\left\|g_{k-1}\right\|^{2}} \\
& <\frac{2\left\|g_{k}\right\|^{2}}{\left\|g_{k-1}\right\|^{2}} .
\end{aligned}
$$

And if

$$
\beta_{k}^{\mathrm{PRP}^{* *}}=\beta_{k}^{\mathrm{NPRP}}=\frac{\left\|g_{k}\right\|^{2}-\left(\left\|g_{k}\right\| /\left\|g_{k-1}\right\|\right)\left|g_{k}^{T} g_{k-1}\right|}{\left\|g_{k-1}\right\|^{2}},
$$

we obtain

$$
0 \leq \beta_{k}^{\mathrm{PRP}^{* *}}<\frac{\left\|g_{k}\right\|^{2}}{\left\|g_{k-1}\right\|^{2}}=\beta_{k}^{\mathrm{FR}}
$$

In Case B there is no guarantee that this method will satisfy the sufficient descent condition.

For the next discussion, we will discuss the nonnegative PRP method which is given as follows:

$$
\begin{aligned}
& \beta_{k}^{\mathrm{PRP}+}=\max \left\{\beta_{k}^{\mathrm{PRP}}, 0\right\} \text { i.e. } \\
& \beta_{k}^{\mathrm{PRP}+}=\max \{\text { Case A1, Case A2, Case B, } 0\} .
\end{aligned}
$$

Therefore, we have a problem in Case B. To solve this problem Gilbert and Nocedal [16] used another line search to satisfy the convergence properties. In addition, if $\beta_{k}^{\mathrm{PRP}+}=0$, the CG method returns to the steepest descent method which is sometimes a weak tool to find the optimum point for functions. Furthermore we can notice that

$$
\beta_{k}^{\mathrm{TS}}= \begin{cases}\text { Case Al } & \\ \beta_{k}^{\mathrm{FR}}, & \text { otherwise. }\end{cases}
$$

So from PRP method we can use only Case Al.

To improve the above ideas, we suggest the following hybrid method:

One of the advantages for $\beta_{k}^{\mathrm{PRP}^{* *}}$ is that we can use Case Al and Case B under the condition $\left\|g_{k}\right\|^{2}>\left|g_{k}^{T} g_{k-1}\right|$.

Choosing $\beta_{k}^{\mathrm{NPRP}}$ as a restart CG formula in (20), if the condition $\left\|g_{k}\right\|^{2}>\left|g_{k}^{T} g_{k-1}\right|$ is not satisfied that means

$$
\left\|g_{k}\right\|^{2} \leq\left|g_{k}^{T} g_{k-1}\right| .
$$

Thus, NPRP method is a suitable nonnegative value to use.

The following algorithm is an algorithm of CG method with the new coefficient $\beta_{k}^{\text {PRP }^{* *}}$.

Algorithm 1. Consider the following.

Step 1. Initialization: given $x_{1}$, set $k=1$.

Step 2. Compute $\beta_{k}$ based on (20).

Step 3. Compute $d_{k}$ based on (3).

Step 4. Compute $\alpha_{k}$ based on (5) and (6).

Step 5. Update new point based on (2).

Step 6. Convergent test and stopping criteria: if $\left\|g_{k}\right\| \leq \varepsilon$ then stop; otherwise go to Step 2 with $k=k+1$. 


\section{The Global Convergence Properties for $\beta_{k}^{\mathrm{PRP}^{* *}}$ with SWP Line Search}

The following standard assumptions are necessary for this work.

Assumption 1. The level set $\mathrm{X}=\left\{x \in R^{n}: f(x) \leq f\left(x_{1}\right)\right\}$, with $x_{1}$ to the starting point of the iterative method (2), is bounded.

Assumption 2. In some open convex neighborhood $N$ of $\mathrm{X}, f$ is continuous and differentiable, and its gradient is Lipschitz continuous; that is, for any $x, y \in N$, there exists a constant $L>0$ such that $\|g(x)-g(y)\| \leq L\|x-y\|$.

The following lemma is one of the most important lemmas which is used to prove the global convergence properties.

Lemma 2 (see [12]). Suppose Assumptions 1 and 2 are true. Consider any form of (2) and (3), with $\alpha_{k}$ computed by WWP line search direction $d_{k}$, is descent for all $k \geq 1$; then

$$
\sum_{k=1}^{\infty} \cos ^{2} \theta_{k}\left\|g_{k}\right\|^{2}<\infty
$$

where

$$
\cos \theta_{k}=-\frac{\left(g_{k}^{T} d_{k}\right)}{\left\|d_{k}\right\|\left\|g_{k}\right\|} .
$$

Equation (25) is known as Zoutendijk condition.

The following discussion will discuss the global convergence properties for $\beta_{k}^{\mathrm{PRP}^{* *}}$ with SWP line search.

Case $1\left(\beta_{k}^{\mathrm{PRP}^{* *}}=\beta_{k}^{\mathrm{NPRP}}\right)$. Since $0 \leq \beta_{k}^{\mathrm{NPRP}} \leq \beta_{k}^{\mathrm{FR}}$ the proof of the global convergence properties is similar to $\beta_{k}^{\mathrm{FR}}$. We refer the reader to see $[13,14]$.

Case $2\left(\beta_{k}^{\mathrm{PRP}^{* *}}=\beta_{k}^{\mathrm{PRP}}\right.$, where $\left.\left\|g_{k}\right\|^{2}>\left|g_{k}^{T} g_{k-1}\right|\right)$. In this case we have

$$
\begin{aligned}
0 & \leq \beta_{k}^{\mathrm{PRP}^{* *}}=\frac{\left\|g_{k}\right\|^{2}-g_{k}^{T} g_{k-1}}{\left\|g_{k-1}\right\|^{2}} \leq \frac{\left\|g_{k}\right\|^{2}+\left|g_{k}^{T} g_{k-1}\right|}{\left\|g_{k-1}\right\|^{2}} \\
& \leq \frac{2\left\|g_{k}\right\|^{2}}{\left\|g_{k-1}\right\|^{2}} .
\end{aligned}
$$

The following theorem demonstrates $\beta_{k}^{\mathrm{PRP}^{* *}}$ in Case 2 satisfies the sufficient descent condition with SWP line search.

Theorem 3. Consider the sequences $g_{k}$ and $d_{k}$ are constructed by Algorithm 1 and $\alpha_{k}$ is computed by (5) and (6) if $\sigma \in$ (0,1/4]. Then (15) holds.

Proof. From (3), for $k=1$, it is obvious. Suppose it is true until $k-1$; that is, $g_{k-1}^{T} d_{k-1}=-\left\|g_{k-1}\right\|^{2}$. Multiply (3) by $g_{k}$; we obtain

$$
g_{k}^{T} d_{k}=g_{k}^{T}\left(-g_{k}^{T}+\beta_{k} d_{k-1}\right)=-\left\|g_{k}\right\|^{2}+\beta_{k} g_{k}^{T} d_{k-1} .
$$

Dividing both sides by $\left\|g_{k-1}\right\|^{2}$, and by using Case 2 and (6), then we obtain

$$
-1+2 \sigma \frac{g_{k-1}^{T} d_{k-1}}{\left\|g_{k-1}\right\|^{2}} \leq \frac{g_{k}^{T} d_{k}}{\left\|g_{k}\right\|^{2}} \leq-1-2 \sigma \frac{g_{k-1}^{T} d_{k-1}}{\left\|g_{k-1}\right\|^{2}} .
$$

From (29) we obtain

$$
-\sum_{j=0}^{k-1}(2 \sigma)^{j} \leq \frac{g_{k}^{T} d_{k}}{\left\|g_{k}\right\|^{2}} \leq-2+\sum_{j=0}^{k-1}(2 \sigma)^{j} .
$$

Since

$$
\sum_{j=0}^{k-1}(2 \sigma)^{j} \leq \frac{1-(2 \sigma)^{k}}{1-2 \sigma}
$$

we have

$$
-\frac{1-(2 \sigma)^{k}}{1-2 \sigma} \leq \frac{g_{k}^{T} d_{k}}{\left\|g_{k}\right\|^{2}} \leq-2+\frac{1-(2 \sigma)^{k}}{1-2 \sigma} .
$$

If $\sigma \leq 1 / 4$ we get

$$
\frac{1-(2 \sigma)^{k}}{1-2 \sigma}<2
$$

Let

$$
c=2-\frac{1-(\sigma)^{k}}{1-\sigma}
$$

then

$$
c-2 \leq \frac{g_{k}^{T} d_{k}}{\left\|g_{k}\right\|^{2}} \leq-c .
$$

Thus

$$
g_{k}^{T} d_{k} \leq-c\left\|g_{k}\right\|^{2},
$$

where $c \in(0,1)$. The proof is complete.

Gilbert and Nocedal [16] presented an important theorem (Theorem 4) to find the global convergence properties for nonnegative PRP method if the descent condition is satisfied. Furthermore [16] presented nice property called Property* as follows.

Property ${ }^{*}$. Consider a CG method of forms (2) and (3), and suppose $0<\gamma \leq\left\|g_{k}\right\| \leq \bar{\gamma}$; we say that the CG method possesses Property ${ }^{*}$ if there exists constant $b>1$, and $\lambda>0$, such that, for all $k \geq 1$, we get $\left|\beta_{k}\right| \leq b$, and if $\left\|x_{k}-x_{k-1}\right\| \leq \lambda$, we obtain

$$
\left|\beta_{k}\right| \leq \frac{1}{2 b}
$$

Theorem 4. Consider any CG method of forms (2) and (3) achieves the following properties:

(I) $\beta_{k} \geq 0$.

(II) The sufficient descent condition (15) holds. 
(III) The Zoutendijk condition (25) is satisfied by the line search.

(IV) Property* holds.

(V) Assumptions 1 and 2 hold.

Then the iterates are globally convergent.

The next lemma shows that if the gradients are bounded away from zero and Property ${ }^{*}$ holds, then a certain fraction of steps cannot be too small. The proof is given in [16]. However, we state it for readability.

Lemma 5. Consider a CG algorithm as defined in (2) and (3) with the parameter $\beta_{k}^{\mathrm{PRP}^{* *}}$. If Assumptions 1 and 2 are satisfied, then Property* holds.

Proof. Let $b=2 \bar{\gamma}^{2} / \gamma^{2} \geq 1$ and $\lambda \leq \gamma^{2} / 2 L \bar{\gamma} b$ :

$$
\begin{aligned}
\left|\beta_{k}^{\mathrm{PRP}^{* *}}\right| & \leq\left|\frac{\left\|g_{k}\right\|^{2}-g_{k}^{T} g_{k-1}}{\left\|g_{k-1}\right\|^{2}}\right| \leq \frac{\left\|g_{k}\right\|^{2}+\left|g_{k}^{T} g_{k-1}\right|}{\left\|g_{k-1}\right\|^{2}} \\
& \leq \frac{\left\|g_{k}\right\|\left(\left\|g_{k}\right\|+\left\|g_{k-1}\right\|\right)}{\left\|g_{k-1}\right\|^{2}} \leq \frac{2 \bar{\gamma}^{2}}{\gamma^{2}}=b>1 .
\end{aligned}
$$

Using $\left\|s_{k}\right\| \leq \lambda$, we have

$$
\left|\beta_{k}^{\mathrm{PRP}^{* *}}\right| \leq\left|\frac{g_{k}^{T}\left(g_{k}-g_{k-1}\right)}{\left\|g_{k-1}\right\|^{2}}\right| \leq \frac{\left\|g_{k}\right\|\left\|g_{k}-g_{k-1}\right\|}{\left\|g_{k-1}\right\|^{2}} .
$$

By Assumption 2,

$$
\left|\beta_{k}^{\mathrm{PRP}^{* *}}\right| \leq \frac{L \lambda \bar{\gamma}}{\gamma^{2}} \leq \frac{1}{2 b}
$$

The proof is complete.

Lemma 6. The CG formula presented in Case 2 has the following properties:

(1) $\beta_{k}^{\mathrm{PRP}^{* *}}>0$, since the condition $\left\|g_{k}\right\|^{2}>\left|g_{k}^{T} g_{k-1}\right|$ forces the CG formula in (20) to be nonnegative.

(2) $\beta_{k}^{\mathrm{PRP}^{* *}}$ satisfies Property ${ }^{*}$, based on Lemma 5.

(3) $\beta_{k}^{\mathrm{PRP}^{* *}}$ satisfies the sufficient descent condition based on Theorem 3 and $c \in(0,1)$.

By using Theorems 3 and 4 and Lemma 6 we have the following convergence result. The proof is similar to Theorem 4.3 which is presented in [16].

Theorem 7. Suppose that Assumption 1 holds. Consider the CG method of forms (2) and (3) and $\beta_{k}$ as in Case 2, where $\alpha_{k}$ is computed by (5) and (6) with $\sigma \leq 1 / 4$; then $\lim _{k \rightarrow \infty}$ inf $\left\|g_{k}\right\|=0$.

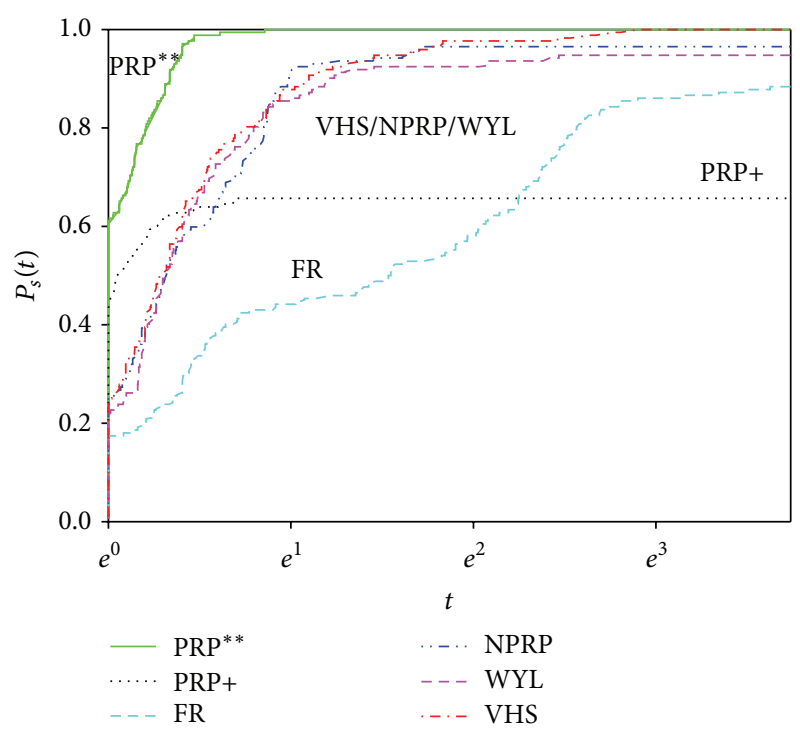

FIGURE 1: Performance profile based on the number of iterations.

\section{Numerical Results and Discussions}

To evaluate the efficiency of the new method, we selected some of the test functions in Table 1 from CUTEr [24], Neculai [23], and Adorio and Diliman [25]. We performed a comparison with other CG methods, including VHS, NPRP, $\mathrm{PRP}+, \mathrm{FR}$, and $\mathrm{PRP}^{* *}$ formulas. The tolerance $\varepsilon$ is selected to $10^{-6}$ for all algorithms to investigate the rapidity of the iteration methods towards the optimal solution. The gradient value is used as the stopping criteria. Here, the stopping criteria considered $\left\|g_{k}\right\| \leq 10^{-6}$. We considered the method failed if the number of iterations exceeds 1000 times.

In Table 1 we selected different initial points for every function. Thus, this demonstrated that this method can be used in several real life functions from other fields such as engineering and medical science as we mentioned before. In addition different dimensions from 500 until 10000 are used. We also choose from different group of functions. We used Matlab 7.9 subroutine program, with CPU processor Intel (R) Core (TM), i3 CPU, and 2 GB DDR2 RAM under SWP line search to find the optimum point. The efficiency comparisons results are shown in Figures 1 and 2, respectively, using a performance profile introduced by Dolan and Moré [26].

This performance measure was introduced to compare a set of solvers $S$ on a set of problems $P$. Assuming $n_{s}$ solvers and $n_{p}$ problems in $S$ and $P$, respectively, the measure $t_{p, s}$ is defined as the computation time (e.g., the number of iterations or the CPU time) required to solve problem $p$ by solver $s$.

To create a baseline for comparison, the performance of solver $s$ on problem $p$ is scaled by the best performance of any solver in $S$ on the problem using the ratio

$$
r_{p, s}=\frac{t_{p, s}}{\min \left\{t_{p, s}: s \in S\right\}} .
$$


TABLE 1: A list of problem functions used with the SWP condition with $\delta=0.01$ and $\sigma=0.1$.

\begin{tabular}{|c|c|c|c|}
\hline Number & Function & Dimension/s & Initial points \\
\hline 1 & $\begin{array}{l}\text { Extended White \& Holst } \\
\text { function [23] }\end{array}$ & $500,1000,5000,10000$ & $(-1.2,1,-1.2,1, \ldots,-1.2,1),(5,5, \ldots, 5),(10,10, \ldots, 10),(15,15, \ldots, 15)$ \\
\hline 2 & $\begin{array}{l}\text { Extended Rosenbrock } \\
\text { function [24] }\end{array}$ & $500,1000,5000,10000$ & $(-1.2,1,-1.2,1, \ldots,-1.2,1),(5,5, \ldots, 5),(10,10, \ldots, 10),(15,15, \ldots, 15)$ \\
\hline 3 & Six-hump function [25] & 2 & $(1,1),(5,5),(10,10),(15,15)$ \\
\hline 4 & $\begin{array}{l}\text { Extended Beale function } \\
\text { [23] }\end{array}$ & $500,1000,5000,10000$ & $(-1,-1, \ldots,-1),(0.5,0.5, \ldots, 0.5),(10,10, \ldots, 10),(1,1, \ldots, 1)$ \\
\hline 5 & Three-hump function [25] & 2 & $(1,1),(5,5),(10,10),(15,15)$ \\
\hline 6 & $\begin{array}{l}\text { Extended Himmelblau } \\
\text { function [23] }\end{array}$ & $500,1000,5000,10000$ & $(1,1, \ldots, 1),(5,5, \ldots, 5),(10,10, \ldots, 10),(15,15, \ldots, 15)$ \\
\hline 7 & Diagonal 2 function [23] & $500,1000,5000,10000$ & $(0.2,0.2, \ldots, 0.2),(0.25,0.25, \ldots, 0.25),(0.5,0.5, \ldots, 0.5),(1,1, \ldots, 1)$ \\
\hline 8 & NONSCOMP function [24] & $500,1000,5000,10000$ & $(1,1, \ldots, 1),(-1,-1, \ldots,-1),(-2,-2, \ldots,-2),(-5,-5, \ldots,-5)$ \\
\hline 9 & $\begin{array}{l}\text { Extended DENSCHNB } \\
\text { function [24] }\end{array}$ & $500,1000,5000,10000$ & $(1,1, \ldots, 1),(5,5, \ldots, 5),(10,10, \ldots, 10),(15,15, \ldots, 15)$ \\
\hline 10 & Booth function $[25]$ & 2 & $(1,1),(5,5),(10,10),(15,15)$ \\
\hline 11 & $\begin{array}{l}\text { Extended quadratic penalty } \\
\text { function QP2 [24] }\end{array}$ & $100,200,300,400$ & $(2,2, \ldots, 2),(5,5, \ldots, 5),(10,10, \ldots, 10),(15,15, \ldots, 15)$ \\
\hline 12 & DIXMAANA function [24] & $1500,3000,6000,9000$ & $(1,1, \ldots, 1),(2,2, \ldots, 2),(3,3, \ldots, 3),(5,5, \ldots, 5)$ \\
\hline 13 & DIXMAANB function [24] & $1500,3000,6000,9000$ & $(1,1, \ldots, 1),(2,2, \ldots, 2),(3,3, \ldots, 3),(5,5, \ldots, 5)$ \\
\hline 14 & DIXMAANC function [24] & $1500,3000,6000,9000$ & $(1,1, \ldots, 1),(2,2, \ldots, 2),(3,3, \ldots, 3),(5,5, \ldots, 5)$ \\
\hline 15 & DIXMAAND function [24] & $1500,3000,6000,9000$ & $(1,1, \ldots, 1),(2,2, \ldots, 2),(3,3, \ldots, 3),(5,5, \ldots, 5)$ \\
\hline 16 & DIXMAANE function [24] & $1500,3000,6000,9000$ & $(1,1, \ldots, 1),(2,2, \ldots, 2),(3,3, \ldots, 3),(5,5, \ldots, 5)$ \\
\hline 17 & DIXMAANF function [24] & $1500,3000,6000,9000$ & $(1,1, \ldots, 1),(2,2, \ldots, 2),(3,3, \ldots, 3),(5,5, \ldots, 5)$ \\
\hline 18 & DIXMAANG function [24] & $1500,3000,6000,9000$ & $(1,1, \ldots, 1),(2,2, \ldots, 2),(3,3, \ldots, 3),(5,5, \ldots, 5)$ \\
\hline 19 & NONDIA function [24] & $500,1000,5000,10000$ & $(-2,-2, \ldots,-2),(-1,-1, \ldots,-1),(0,0, \ldots, 0),(1,1, \ldots, 1)$ \\
\hline 20 & $\begin{array}{l}\text { Extended Tridiagonal } 1 \\
\text { function [24] }\end{array}$ & $500,1000,5000,10000$ & $(1,1, \ldots, 1),(5,5, \ldots, 5),(10,10, \ldots, 10),(15,15, \ldots, 15)$ \\
\hline 21 & DQDRTIC function [24] & $500,1000,5000,10000$ & $(-1,-1, \ldots,-1),(1,1, \ldots, 1),(2,2, \ldots, 2),(3,3, \ldots, 3)$ \\
\hline 22 & Diagonal 4 function $[24]$ & $500,1000,5000,10000$ & $(1,1, \ldots, 1),(5,5, \ldots, 5),(10,10, \ldots, 10),(15,15, \ldots, 15)$ \\
\hline 23 & DQDRTIC function [24] & $10,20,30,40$ & $(1,1, \ldots, 1),(-1,-1, \ldots,-1),(1,1, \ldots, 1),(2,2, \ldots, 2)$ \\
\hline 24 & $\begin{array}{l}\text { Extended Cliff function } \\
{[24]}\end{array}$ & $100,200,300,400$ & $(1,1, \ldots, 1),(5,5, \ldots, 5),(10,10, \ldots, 10),(15,15, \ldots, 15)$ \\
\hline
\end{tabular}

Suppose that a parameter $r_{M} \geq r_{p, s}$ for all $p, s$ is chosen, and $r_{p, s}=r_{M}$ if and only if solver $s$ does not solve problem $p$. Because we would like to obtain an overall assessment of the performance of a solver, we defined the measure

$$
\rho_{s}(t)=\frac{1}{n_{p}} \text { size }\left\{p \in P: r_{p, s} \leq t\right\} .
$$

Thus, $\rho_{s}(t)$ is the probability for solver $s \in S$ that the performance ratio $r_{p, s}$ is within a factor $t \in R$ of the best possible ratio. If we define the function $p_{s}$ as the cumulative distribution function for the performance ratio, then the performance measure $p_{s}: R \rightarrow[0,1]$ for a solver is nondecreasing and piecewise continuous from the right. The value of $p_{s}(1)$ is the probability that the solver has the best performance of all of the solvers. In general, a solver with high values of $p(t)$, which would appear in the upper right corner of the figure, is preferable.

Based on the left side of Figures 1 and 2 the PRP** formula is above the other curves. Therefore, it is the most efficient method among related PRP methods in terms of efficiency and robustness. In Figure 2 we see that the curve of $\mathrm{PRP}^{* *}$ is still the best, but the efficiency is not good as the number of iterations since we use the complicated hybrid algorithm leads to high CPU time. Thus, using high processors computers to find the solution will be more efficient since the number of iterations decreased rapidly under PRP ${ }^{* *}$ method.

\section{Conclusion}

In this paper, we proposed hybrid conjugate gradient method by using nonnegative PRP and NPRP formulas with the SWP line search which extended the cases of using PRP method under mild condition. The global convergence property is established and it is very simple. Our numerical results had shown that the hybrid method is the best when compared to other related PRP CG methods. 


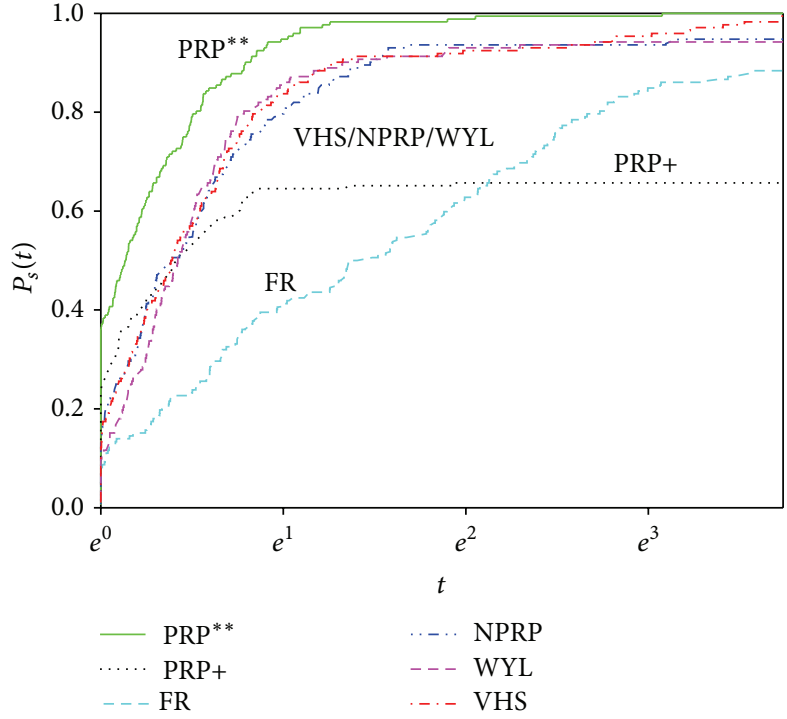

Figure 2: Performance profile based on the CPU time.

\section{Conflict of Interests}

The authors declare that there is no conflict of interests regarding the publication of this paper.

\section{Acknowledgments}

The authors are grateful to the editor and the anonymous reviewers for their comments and suggestions which improved this paper substantially. They would also like to thank The Ministry of Education Malaysia (MOE) for funding this research under The Fundamental Research Grant Scheme (Grant no. 59256).

\section{References}

[1] P. Wolfe, "Convergence conditions for ascent methods," SIAM Review, vol. 11, no. 2, pp. 226-235, 1969.

[2] P. Wolfe, "Convergence conditions for ascent methods. II: some corrections," SIAM Review, vol. 13, no. 2, pp. 185-188, 1971.

[3] J. Cai, Q. Li, L. Li, H. Peng, and Y. Yang, "A fuzzy adaptive chaotic ant swarm optimization for economic dispatch," International Journal of Electrical Power and Energy Systems, vol. 34, no. 1, pp. 154-160, 2012.

[4] K. Natallia, An Introduction to Heuristic Slgorithms, Department of Informatics and Telecommunications, 2005.

[5] L. Li, H. Peng, J. Kurths, Y. Yang, and H. J. Schellnhuber, "Chaosorder transition in foraging behavior of ants," Proceedings of the National Academy of Sciences of the United States of America, vol. 111, no. 23, pp. 8392-8397, 2014.

[6] M. Wan, L. Li, J. Xiao, C. Wang, and Y. Yang, "Data clustering using bacterial foraging optimization," Journal of Intelligent Information Systems, vol. 38, no. 2, pp. 321-341, 2012.

[7] M. Wan, C. Wang, L. Li, and Y. Yang, "Chaotic ant swarm approach for data clustering," Applied Soft Computing Journal, vol. 12, no. 8, pp. 2387-2393, 2012.
[8] M. R. Hestenes and E. Stiefel, "Methods of conjugate gradients for solving linear systems," Journal of Research of the National Bureau of Standards, vol. 49, no. 6, pp. 409-436, 1952.

[9] R. Fletcher and C. M. Reeves, "Function minimization by conjugate gradients," The Computer Journal, vol. 7, no. 2, pp. 149-154, 1964.

[10] P. Elijah and G. Ribiere, "Note sur la convergence de méthodes de directions conjuguées," Revue française d'informatique et de recherche opérationnelle, vol. 3, no. 1, pp. 35-43, 1969.

[11] Z. Wei, S. Yao, and L. Liu, “The convergence properties of some new conjugate gradient methods," Applied Mathematics and Computation, vol. 183, no. 2, pp. 1341-1350, 2006.

[12] G. Zoutendijk, "Nonlinear programming, computational methods," Integer and Nonlinear Programming, vol. 143, no. 1, pp. 37$86,1970$.

[13] M. Al-Baali, "Descent property and global convergence of the Fletcher-Reeves method with inexact line search," IMA Journal of Numerical Analysis, vol. 5, no. 1, pp. 121-124, 1985.

[14] L. Guanghui, H. Jiye, and Y. Hongxia, "Global convergence of the fletcher-reeves algorithm with inexact linesearch," Applied Mathematics-A Journal of Chinese Universities, vol. 10, no. 1, pp. 75-82, 1995.

[15] M. J. D. Powell, "Nonconvex minimization calculations and the conjugate gradient method," in Numerical Analysis, vol. 1066 of Lecture Notes in Mathematics, pp. 122-141, Springer, Berlin, Germany, 1984.

[16] J. C. Gilbert and J. Nocedal, "Global convergence properties of conjugate gradient methods for optimization," SIAM Journal on Optimization, vol. 2, no. 1, pp. 21-42, 1992.

[17] D. Touati-Ahmed and C. Storey, "Efficient hybrid conjugate gradient techniques," Journal of Optimization Theory and Applications, vol. 64, no. 2, pp. 379-397, 1990.

[18] Y. Shengwei, Z. Wei, and H. Huang, "A note about WYL's conjugate gradient method and its applications," Applied Mathematics and Computation, vol. 191, no. 2, pp. 381-388, 2007.

[19] L. Zhang, "An improved Wei-Yao-Liu nonlinear conjugate gradient method for optimization computation," Applied Mathematics and Computation, vol. 215, no. 6, pp. 2269-2274, 2009.

[20] Z. Dai and F. Wen, "Another improved Wei-Yao-Liu nonlinear conjugate gradient method with sufficient descent property," Applied Mathematics and Computation, vol. 218, no. 14, pp. 74217430, 2012.

[21] M. Rivaie, M. Mamat, L. W. June, and I. Mohd, "A new class of nonlinear conjugate gradient coefficients with global convergence properties," Applied Mathematics and Computation, vol. 218, no. 22, pp. 11323-11332, 2012.

[22] A. Alhawarat, M. Mamat, M. Rivaie, and I. Mohd, "A new modification of nonlinear conjugate gradient coefficients with global convergence properties," International Journal of Mathematical, Computational, Statistical, Natural and Physical Engineering, vol. 8, no. 1, pp. 54-60, 2014.

[23] A. Neculai, "An unconstrained optimization test functions collection," Advanced Modeling and Optimization, vol. 10, no. 1, pp. 147-161, 2008.

[24] I. Bongartz, A. R. Conn, N. Gould, P. L. Toint, and I. Bongartz, Constrained and Unconstrained Testing Environment, Département de Mathématique, 1993.

[25] E. P. Adorio and U. Diliman, "Mvf-multivariate test functions library in c for unconstrained global optimization," 2005.

[26] E. D. Dolan and J. J. Moré, "Benchmarking optimization software with performance profiles," Mathematical Programming, vol. 91, no. 2, pp. 201-213, 2002. 


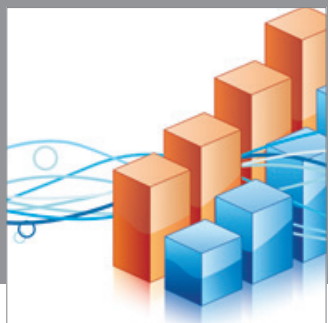

Advances in

Operations Research

mansans

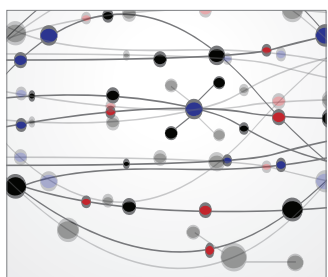

The Scientific World Journal
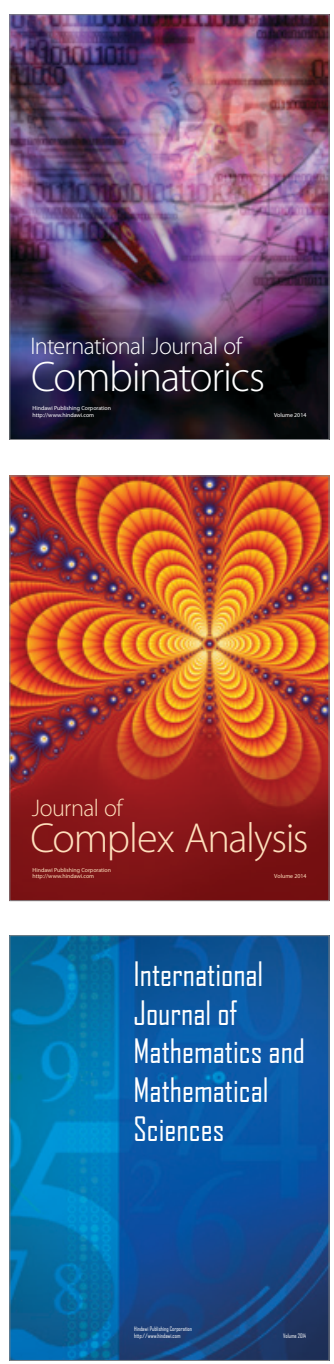
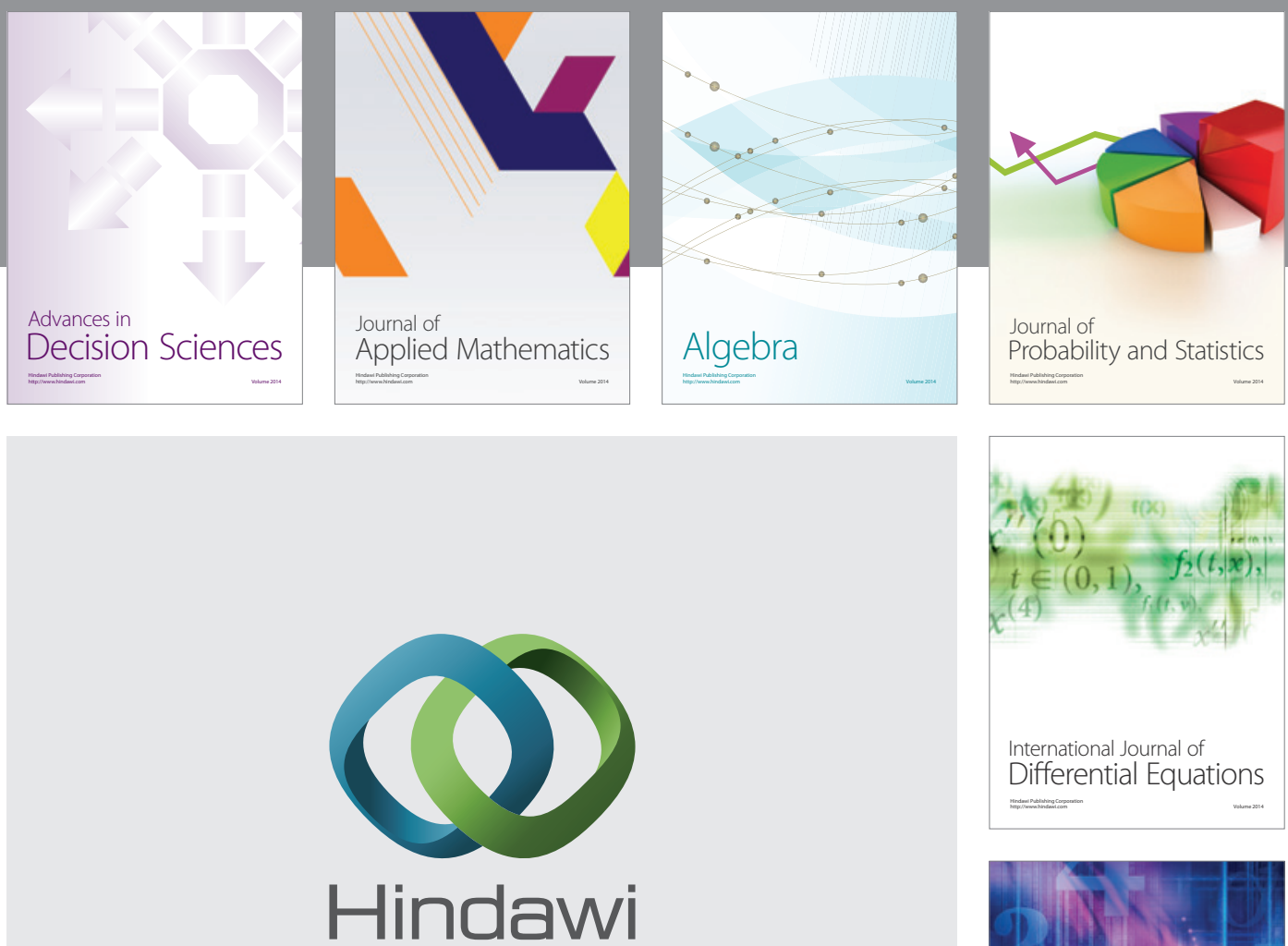

Submit your manuscripts at http://www.hindawi.com
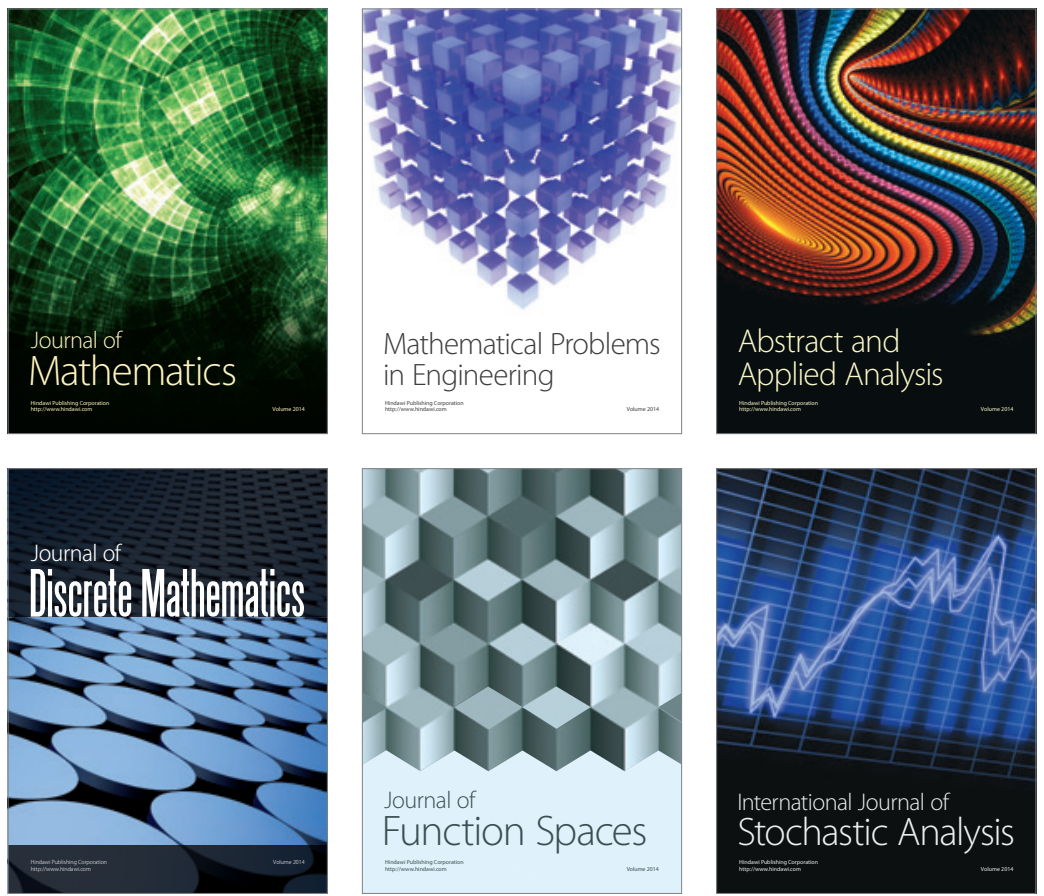

Journal of

Function Spaces

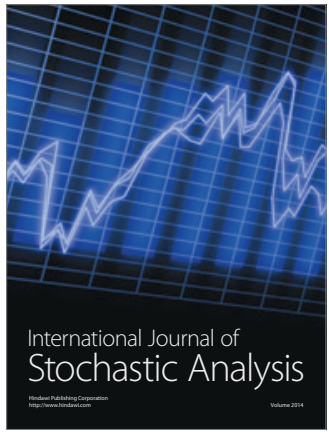

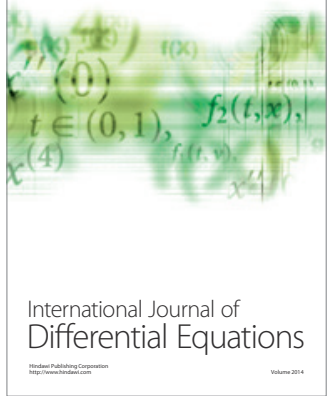
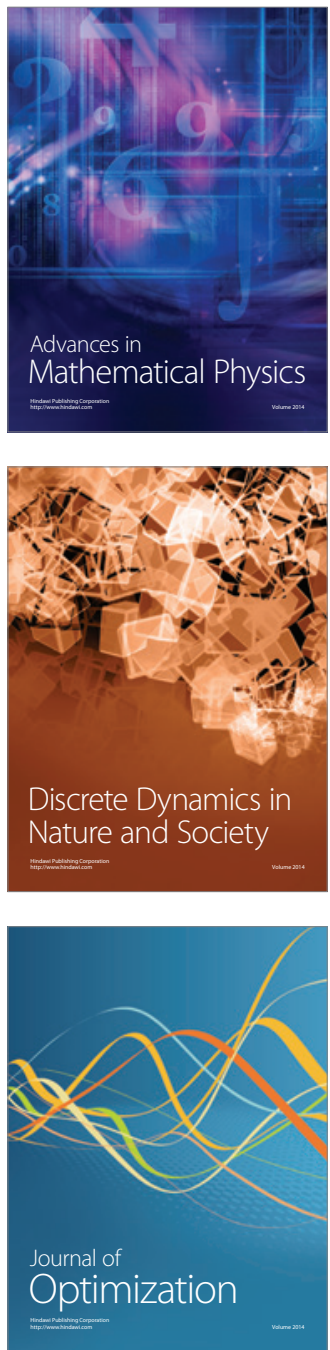